\title{
miR-504 modulates the stemness and mesenchymal transition of glioma stem cells and their interaction with microglia via delivery by extracellular vesicles
}

\author{
Ariel Bier ${ }^{1}$, Xin Hong ${ }^{2}$, Simona Cazacu², Hodaya Goldstein ${ }^{2}$, Daniel Rand ${ }^{1}$, Cunli Xiang², Wei Jiang ${ }^{2}$, \\ Hiba Waldman Ben-Asher ${ }^{1}$, Moshe Attia ${ }^{3}$, Aharon Brodie', Ruicong She ${ }^{4}$, Laila M. Poisson ${ }^{4}$ and Chaya Brodie ${ }^{1,2}$
}

\begin{abstract}
Glioblastoma (GBM) is a highly aggressive tumor with poor prognosis. A small subpopulation of glioma stem cells (GSCs) has been implicated in radiation resistance and tumor recurrence. In this study we analyzed the expression of miRNAs associated with the functions of GSCs using miRNA microarray analysis of these cells compared with human neural stem cells. These analyses identified gene clusters associated with glioma cell invasiveness, axonal guidance, and TGF- $\beta$ signaling. miR-504 was significantly downregulated in GSCs compared with NSCs, its expression was lower in GBM compared with normal brain specimens and further decreased in the mesenchymal glioma subtype.

Overexpression of miR-504 in GSCs inhibited their self-renewal, migration and the expression of mesenchymal markers. The inhibitory effect of miR-504 was mediated by targeting Grb10 expression which acts as an oncogene in GSCs and GBM. Overexpression of exogenous miR-504 resulted also in its delivery to cocultured microglia by GSC-secreted extracellular vesicles (EVs) and in the abrogation of the GSC-induced polarization of microglia to M2 subtype. Finally, miR-504 overexpression prolonged the survival of mice harboring GSC-derived xenografts and decreased tumor growth. In summary, we identified miRNAs and potential target networks that play a role in the stemness and mesenchymal transition of GSCs and the miR-504/Grb10 pathway as an important regulator of this process. Overexpression of miR-504 exerted antitumor effects in GSCs as well as bystander effects on the polarization of microglia via delivery by EVs.
\end{abstract}

\section{Introduction}

Glioblastomas (GBMs) are the most common and aggressive of the astrocytic tumors. They are characterized by increased proliferation and angiogenesis, invasion into the surrounding normal tissue and resistance to

\footnotetext{
Correspondence: Chaya Brodie (chaya@brodienet.com)

${ }^{1}$ The Mina and Everard Goodman Faculty of Life Sciences, Bar-llan University, Ramat-Gan, Israel

2Davidson Laboratory of Cell Signaling and Tumorigenesis, Hermelin Brain Tumor Center, Department of Neurosurgery, Henry Ford Hospital, Detroit, $\mathrm{Ml}$, USA

Full list of author information is available at the end of the article These authors contributed equally: Ariel Bier, Xin Hong, Simona Cazacu Edited by $\mathrm{G}$. Calin
}

therapies ${ }^{1}$. The prognosis of patients with GBM remains extremely poor, and the median survival of GBM patients has remained around 14-16 months over the past dec$\operatorname{ades}^{2}$. Gene expression profiling studies identified GBM subtypes that are classified based on their transcriptional signatures into various molecular groups, including proneural, classical, and mesenchymal ${ }^{3,4}$. Recently, the profiling of DNA methylation patterns in glioma has refined these categories ${ }^{5}$, aligning them with the WHO 2016 diagnostic schema for glioma. These subtypes have distinct differential genetic alterations, molecular signatures, cellular phenotypes, and patient prognosis ${ }^{5-7}$.

\section{(c) The Author(s) 2020}

(c) (i) Open Access This article is licensed under a Creative Commons Attribution 4.0 International License, which permits use, sharing, adaptation, distribution and reproduction cc) in any medium or format, as long as you give appropriate credit to the original author(s) and the source, provide a link to the Creative Commons license, and indicate if changes were made. The images or other third party material in this article are included in the article's Creative Commons license, unless indicated otherwise in a credit line to the material. If material is not included in the article's Creative Commons license and your intended use is not permitted by statutory regulation or exceeds the permitted use, you will need to obtain permission directly from the copyright holder. To view a copy of this license, visit http://creativecommons.org/licenses/by/4.0/. 
GBMs contain a small subpopulation of cancer stem cells (i.e., glioma stem cells [GSCs] $)^{8}$ that are characterized by self-renewal, multi-lineage differentiation potential, and the ability to generate xenografts that recapitulate the parental tumors ${ }^{9}$. GSCs have been implicated in tumor infiltration and resistance to radio- and chemotherapy as well as tumor recurrence ${ }^{10}$. GSCs share stemness characteristics with neural stem cells (NSCs) but differ in their differentiation and oncogenic potentials ${ }^{9,11,12}$.

The epithelial-to-mesenchymal transition (EMT) is a process that allows epithelial cells to abandon their polarity and cell-to-cell adhesion properties and acquire mesenchymal cell phenotypes which are associated with enhanced invasiveness, stemness and metastasis, and treatment resistance ${ }^{13}$. Recent studies demonstrated a similar process in glioma, and proneural-to-mesenchymal transition in these tumors is characterized by increased aggressiveness, invasiveness and therapy resistance ${ }^{14-16}$. Mesenchymal transition can occur in recurrent tumors and in response to radiation ${ }^{17,18}$ and is associated with poor patient prognosis.

Tumor aggressiveness and mesenchymal transition of glioma are induced by cells and secreted factors in the tumor microenvironment ${ }^{19-21}$. These cells include endogenous central nervous system (CNS) cells such as astrocytes, oligodendrocytes, neurons and microglia, and infiltrating immune cells ${ }^{21}$. Microglia are resident immune cells in the brain and together with infiltrating macrophages represent the most prevalent CNS-associated cells in the tumors $^{22}$. Microglia and recruited macrophages have been reported to undergo differentiation to cells with M2-type characteristics, in response to factors secreted by glioma cells $^{23}$. The M2 microglia and macrophages further support tumor growth via the secretion of growth factors, chemokines and extracellular matrix-modifying enzymes ${ }^{22,24}$. In addition, recent studies implicated extracellular vesicles (EVs) as important mediators of intercellular communication and in the cross talk of tumor cells and their microenvironments ${ }^{25}$. EVs contain proteins, lipids, and various RNA species and play important roles in the interaction of glioma cells and microglia ${ }^{23,25}$.

The expression of specific microRNAs (miRNAs) has been shown to be associated with several aspects of glioma pathogenesis including cell cycle control, invasion, migration, resistance to therapies, and cell apoptosis ${ }^{26}$. Specific miRNAs have been also implicated in the selfrenewal, stemness, and tumorigenic features of $\mathrm{GSCs}^{27}$.

Here, we analyzed the miRNA expression of GSCs in comparison with human NSCs (hNSCs) and identified unique miRNA expression profiles that distinguish these two cell populations. Focusing on miR-504, which was highly expressed in hNSCs compared to GSCs, we demonstrated that it was upregulated in the G-CIMP glioma subtype compared to other GBM subtypes.
Moreover, we found that miR-504 exerted an antitumor effect in vitro and in vivo and in addition, inhibited the stemness and mesenchymal transit of GSCs. In addition, overexpression of miR-504 in GSCs exerted a bystander effect on cocultured microglia cells by promoting the differentiation of these cells toward M1 phenotype via EV delivery.

\section{Materials and methods GSC cultures}

All human materials were used in accordance with the policies of the Henry Ford Hospital Institutional Review Board. Generation of GSCs from fresh GBM specimens and their characterization have been recently described ${ }^{28-31}$. Briefly, the GSCs were maintained in neurosphere medium (DMEM-F12 1:1, glutamine $10 \mathrm{mM}$, HEPES buffer $10 \mathrm{mM}$, and sodium bicarbonate $0.025 \%$ ) supplemented with basic fibroblast growth factor $(20 \mathrm{ng} / \mathrm{ml})$ and epidermal growth factor $(20 \mathrm{ng} / \mathrm{ml})$. The GSCs expressed markers such as CD44, CD133, Musashi-1, Sox2, and nestin, exhibited selfrenewal, and expressed astrocytic and neuronal markers upon differentiation. The GSCs also exhibited tumorigenic potential and generated glioma xenografts in nude mice ${ }^{28-33}$. The full information of the GSCs employed in this study is described in Table S1.

\section{Microglia and NSC cultures}

Human microglial cells were obtained from Applied Biological Material (Richmond, BC, Canada). All cells employed in this study were tested for mycoplasma contamination (Mycoplasma PCR Detection Kit) and found negative. hNSCs (H9, hESC derived) were obtained from Invitrogen.

\section{Transduction of GSCs and microglial cells}

Lentivirus vectors (System Biosciences, Mountain View, CA) expressing the miR-504 reporter, pre-miR-504, miR504 antagomiR, Grb10, or control and Grb10 shRNAs were packaged and used to transduce the cells according to the manufacturer's protocol and as previously described ${ }^{28-30}$.

\section{Neurosphere formation assay}

To determine the ability of GSCs to form secondary neurospheres, cells were plated in 24-well plates at a density of 10 and 100 cells/well through limiting dilution and the number of neurospheres/well was determined following 10 days for ten different wells. Spheres that contained more than 20 cells were scored and the results are presented as percentages of maximal neurospheres formed compared to control cells ${ }^{28,29}$.

\section{In vitro limiting dilution assay}

For the in vitro limiting dilution assay, GSCs were plated in 96-well plates in decreasing numbers of cells (50, 
$20,10,5,2$, and 1) per well. Following 10 days, the number of spheres was determined for each well. Extreme limiting dilution was analyzed as recently reported ${ }^{32}$.

\section{Real-time polymerase chain reaction (RT-PCR)}

Total RNA was extracted using RNeasy midi kit according to the manufacturer's instructions (Qiagen, Frederick, MD). Reverse transcription reaction was carried out using 2- $\mu$ g total RNA as previously described ${ }^{28,31}$. Briefly, reactions were run on an ABI Prism 7000 Sequence Detection System (Applied Biosystems, Foster City, CA). Cycle threshold (Ct) values were obtained from the ABI 7000 software. S12 levels were used as controls. The primer sequences are described in Table S2.

\section{Western Blot analysis}

Cell pellet preparation and Western Blot analyses were performed as previously described ${ }^{28-30}$.

\section{Transwell migration assay}

Transwell chambers (BD Biosciences, San Jose, CA) were used for analyzing cell migration as recently reported ${ }^{29,34}$ In brief, cells $(25,000 /$ well $)$ were incubated for $3 \mathrm{~h}$ in culture medium with $10 \%$ fetal bovine serum in the bottom chambers. The total number of the migrated cells was determined in fixed and stained cells $(0.05 \%$ crystal violet for $5 \mathrm{~min})$.

\section{Cell viability assay}

Cells were washed with phosphate-buffered saline (PBS), centrifugated in $3000 \mathrm{rpm}$ for $5 \mathrm{~min}$ and the cell pellet was incubated in PBS containing 0.4\% trypan to stain the dead cells. The number of Trypan-blue stained cells was determined using a Countess II FL (Thermo Fisher, MA, USA).

\section{Isolation of GSC-derived EVs}

EVs were prepared as previously described ${ }^{31,35}$ using sequential centrifugation at $300 \times g$ for $10 \mathrm{~min}, 2500 \times g$ for $20 \mathrm{~min}, 10,000 \times g$ for $30 \mathrm{~min}$ and $110,000 \times g$ for $90 \mathrm{~min}$. The pellet was then resuspended in PBS and washed twice followed by filtration using a $0.22-\mu \mathrm{m}$ filter. The protein content of the enriched EV fractions was determined using the Micro BCA assay kit (ThermoFischer Scientific, Oregon City, OR). The expression of the exosome markers CD63, CD81, and CD9 was analyzed by Western blot and the quantification of the isolated EVs was performed using the ExoELISA-Ultra CD63 kit according to the manufacturer's instructions. For the exosome treatment, $0.5 \times 10^{8} \mathrm{EVs}$ were added to the cultured cells.

\section{ImageStream $X$ analysis}

Microglial cells were treated with GSC-derived EVs labeled with CellTracker Red (ThermoFisher, Waltham, MA) for $24 \mathrm{~h}$. Cells were excited using 561-nm laser, and cell fluorescence of approximately $10^{4}$ cells per sample was captured and photographed using an ImageStreamX highresolution imaging flow cytometer (Amnis Co., Seattle, WA) as previously described ${ }^{35}$. The samples were gated to obtain a population of captured single-cell images of living cells, then gated for the cells in focus using the gradient root mean square feature. Cells incubated with or without labeled EVs were compared for the intensity of the red channel fluorescence. Images were analyzed using IDEAS 6.0 software (Amnis Co., Seattle, WA).

\section{miR-504 reporter}

For analyzing miR-504 delivery, a miR-504 luciferase reporter plasmid was employed as previously described for miR-124 $4^{36}$. A unique miR-504 binding site, which is a fully complementary sequence of mature miR-504, was cloned downstream of luciferase reporter gene of the pMiR-Luc reporter vector from Signosis, Inc. (Santa Clara, CA). For the mCherry reporter, the luciferase gene of pMiR-Luc reporter vector was replaced with mCherryN1 obtained from Clontech (Mountain View, CA).

\section{Phagocytosis analysis}

Human microglial cells were plated alone or in coculture with GSCs. Phagocytosis was determined using the pHrodo Green zymosan bioparticle assay (Invitrogen, Carlsbad, CA, USA) according to the manufacturer's instructions. Briefly, microglia plated alone and in the presence of GSCs were incubated with a solution of pHrodo Green zymosan bioparticles in Live Cell Imaging Solution $(0.5 \mathrm{mg} / \mathrm{ml})$. Phagocytosis was determined after $120 \mathrm{~min}$ using fluorescence plate reader at Ex/Em 509/533.

\section{miRNA array processing and analysis}

All experiments were performed using Affymetrix $\mathrm{HU}$ GENE1.0st oligonucleotide arrays and GeneChip miRNA 4.0 Array (ThermoFisher). Sample processing was performed according to the protocol provided by the company. The rest of the analysis was performed using Partek ${ }^{\circledR}$ Genomics Suite ${ }^{\mathrm{TM}}$ software, version $6.6\left({ }^{\odot} 2012\right.$ Partek, Inc.). miRNA data were summarized using RMA and standardized by sketch-quantile normalization. Differential expression was performed via ANOVA. Significant miRNAs were selected to have at least 1.5 -fold change and a $P$ value $<0.05$. Results were visualized by volcano plot. Functional analysis was conducted by Ingenuity software using the core analysis on differential miRNA lists. The panel of measured miRNAs (a list of all measured miRNAs) was used as the background set for enrichment tests. Networks included up to 35 miRNAs and mRNAs.

\section{TCGA data analysis}

Expression data were downloaded for TCGA cases from the Broad Firehose portal (http://gdac.broadinstitute.org/). GBM cases were assayed by microarray for miRNA 
expression $^{6}$. The level 3, batch-adjusted, expression data file captured mature miRNA quantification (file date: 12/10/ 2014). Low-grade glioma (LGG) cases were assayed by miRNA-sequencing ${ }^{37}$. The level 3 data file contained expression data per mature miRNA as reads per million miRNAs mapped (file date: 12/10/2014). GBM and LGG cases were assayed by mRNA-sequencing ${ }^{5}$. The level 3 data file contained RSEM normalized data ${ }^{38}$, quantified per-gene as the normalized count (file date: 12/10/2014). Expression data are used continuously, discretized by quantile, or dichotomized at the median as high/low as appropriate for the research question. Clinical data and molecular classifications were taken from the recent publication of the TCGA Glioma Analysis Working Group ${ }^{6}$. Comparison of mean expression between groups was performed by one-way ANOVA followed by Tukey's corrected two-sample tests, which adjust for multiple comparisons to maintain the family-wise error rate.

\section{Xenograft studies}

Following the guidelines of Henry Ford Hospital's Institutional Animal Care and Use Committee, dissociated GSCs $\left(3 \times 10^{5}\right.$ cells $)$ transduced with a lentivirus vector expressing a control pre-miR or pre-miR-504 were inoculated intracranially into nude mice $(\mathrm{Nu} / \mathrm{Nu})$ as previously described ${ }^{32}$. Briefly, animals were anesthetized and injected with the GSCs through a 3- $\mathrm{mm}$ hole to the right of the bregma at a depth of $2.5 \mathrm{~mm}$ and a rate of $5 \mu \mathrm{L} / 30 \mathrm{~s}$. All animals were monitored daily and sacrificed at the first signs of neurological deficit.

\section{Statistical analysis}

The results are presented as the mean values \pm SD. The data of patient specimens are presented graphically with median and interquartile range noted. Data were analyzed using ANOVA or a Student's $t$ test with correction for data sets with unequal variances. Data were analyzed on a $\log 2$ scale as appropriate. Kaplan-Meier estimates of the survival time from diagnosis until death or last follow-up were used for outcome analysis. Differences in survival curves between groups were assessed by the log-rank test. Cox regression was used to construct multivariable models of survival including miRNA expression, age at diagnosis, IDH mutation status and grade.

\section{Results}

\section{Functional clustering and networks associated with miRNAs that distinguish GSCs from hNSCs}

To define the patterns of miRNA expressions that are unique to GSCs and associated with their tumorigenicity and mesenchymal characteristics, we used a miRNA array chip for 12 GSCs and three different cultures of hNSCs. We first compared the miRNA expression of GSCs and hNSCs. miRNAs were identified using cutoffs for $\geq 1.5$ - fold differential expression and a significant $P$ value $(P \leq$ $0.05)$, as listed in Table $S 3$ and as shown in a volcano plot (Fig. 1A). Thirty miRNAs were significantly upregulated, and 55 miRNAs were downregulated in GSCs relative to hNSCs. These miRNAs were further analyzed by functional enrichment and network analysis using Ingenuity Pathway Analysis (IPA; Ingenuity Systems, http://www. ingenuity.com). IPA analysis identified clusters of miRNAs that are associated with well-known oncogenic pathways including cell cycle, cellular development, cellular growth and proliferation, cell-to-cell signaling and interaction and cell death and survival (Fig. 1B). IPA was also used to generate three networks of altered miRNA interactions consisting of at least 15 miRNAs from the miRNA lists (Figs. 1C, S1A, B). These networks are associated with miRNA biogenesis including regulation of Dicer1 and AGO2 (Figs. 1C, S1A) and of oncogenes such as TERT, MYC, CASP2, CASP10, BCL2, and TP73 (Fig. S1a). An important oncogenic pathway that was also identified is associated with increased regulation of classical EMT mediators such as Smad2/3, Smad6/7, TGF $\beta 1$, and Dicer (Figs. 1C, S1B).

We then identified miRNAs that were expressed in GSCs but not in hNSCs and found that 10 miRNAs were significantly upregulated in GSCs while 37 were downregulated (Fig. S1D). The IPA of these miRNA clusters generated two networks, each containing more than 10 miRNAs (Figs. 1D, S1C). These networks consist of proteins related to cell survival (p53 and TERT) and miRNA biogenesis (Dicer and AGO2) similar to the pathways that were obtained in the initial comparison of the GSCs and hNSCs (Fig. 1D). In addition, the two key mesenchymal markers ZEB2 and RUNX1 were also identified in these networks (Fig. S1C).

\section{MiR-504 is downregulated in GBMs and GSCs}

Using RT-PCR analysis we first validated some of the $\mathrm{miR}$ array results (Figs. 2A, S2). Since miR-504 was one of the most downregulated miRNAs in GSCs compared with hNSCs (Fig. 1A), we focused on this miRNA as a potential inhibitor of the tumorigenicity of GSCs. The expression of miR-504 in GBM specimens was also significantly increased in normal brain compared with astrocytic tumor specimens (Fig. 2B).

We then analyzed the relative expression of miR-504 in the different subtypes of GBM using TCGA ${ }^{6}$. There are 339 GBM cases in our study that have IDH/Methylation subtyping. This subtyping splits the IDHmut-noncodel class into two groups according to methylation pattern (Fig. 2C). The G-CMIP-low class has a lower level of methylation globally and has been found to have worse prognosis. The IDHwt GBM tumors are split into three groups. Two align with expression class, as mesenchymallike and classic-like, and the third has a distinct methylation pattern, denoted as LGm6-GBM (Fig. 2C, 


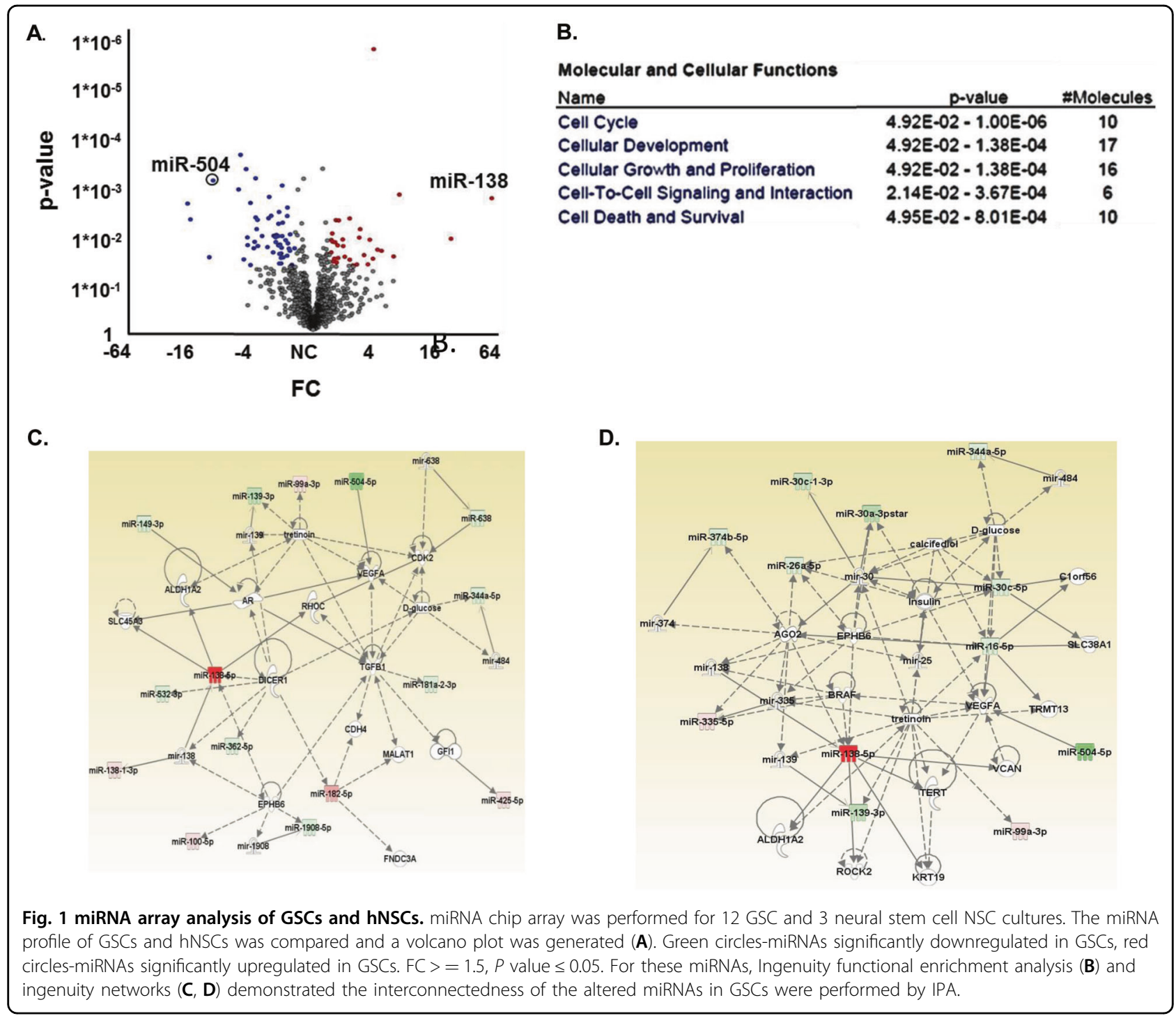

ANOVA, $P<0.0001)$. Gray lines indicate significant difference between groups (post hoc $t$ test, $P<0.05$ ). Analysis of miR-504 expression in LLGs demonstrated that the expression of miR-504 was higher on average in grade II glioma compared with grade III (Fig. 2D).

There are 509 LGG cases in our study that have IDH/ methylation subtyping. This subtyping splits the IDHmut class into three groups according to methylation pattern (Fig. 2E). The G-CMIP-high class has a highest miR-504 expression. The IDHwt LGG tumors are also split into three groups. The classic-like and PA-like are two groups with the lowest and highest expression, respectively. While the mesenchymal-like group has intermediate expression (Fig. 2E). Gray lines indicate significant difference between groups (post hoc $t$ test, $P<0.05$ ). While survival differences were observed by IDH-mutation status (data not shown), there was no evidence that miR-504 expression has independent prognostic value beyond the two new WHO 2016 diagnostic groups, GBM with IDHwt and GBM with IDH mutant. A Kaplan-Meier plot demonstrates that the quarter of patients with lowest expression of miR-504 has the worst survival outcome among grade II and III glioma (Fig. 2F; $\log$-rank $P=$ 0.00136 overall; $\log$-rank $P=0.00402,0.00123,0.0111 \mathrm{Q} 1$ vs. Q2-4, respectively). Collectively, these results indicate that miR-504 expression is inversely correlated with tumor aggressiveness and poor prognosis.

\section{miR-504 inhibits the stemness and mesenchymal transit of GSCs}

To examine the effect of miR-504 we overexpressed it in GSCs (Fig. 3SA) and found that pre-miR-504 markedly decreased the expression of the stemness markers Oct 4 and Nanog and increased the expression of the 

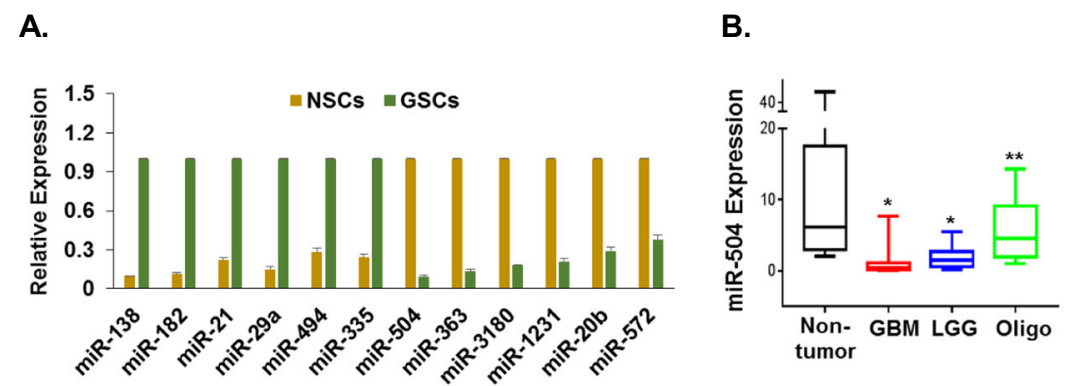

D.

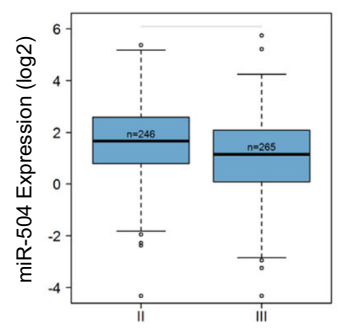

E.

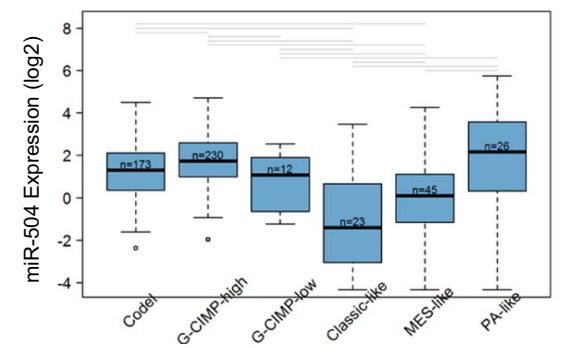

C.

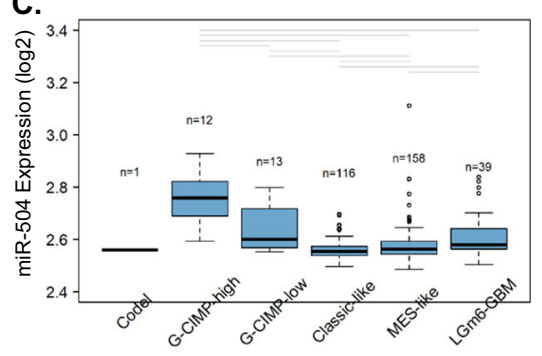

F.

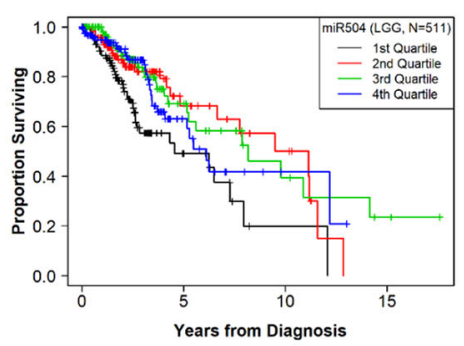

Fig. 2 Expression of miR-504 in GSCs and glial tumors. Validation of top miRNAs enriched in GSCs $(N=11)$ compared with the hNSCs ( $N=4)$ was performed by RT-PCR (A) $P<0.001$. miR-504 expression in glial tumors compared with normal brains $(n=20$ for each) was determined using realtime PCR (B) $P<0.001$. Relative expression of miR-504 in GBM (C) and LGG (E) by subtypes was determined according to The Cancer Genome Atlas (TCGA data portal): gray bars indicate significant differences in post hoc $t$ tests $P<0.05$. Relative expression of miR-504 was also analyzed in grade III and grade II LGG (D). Overall survival plotted according to quartile of miR-504 expression among grade II and III glioma $(\mathbf{F}) ; \log$-rank $P=0.00136$ overall; log-rank $P=0.00402,0.00123,0.0111, Q 1$ vs. Q2-4, respectively.

astrocytic marker GFAP in both GSC-1 and GSC-2 (Fig. 3A).

The role of miR-504 in the stemness of GSCs was further examined on the self-renewal ability and neurosphere formation of these cells. Overexpression of miR-504 in GSCs decreased their ability to form neurospheres as indicated by analyzing secondary neurosphere formation (Fig. 3B), the extreme limiting dilution assay (Fig. 3C) and spheroid size (Fig. 3D), suggesting that miR-504 inhibited the stemness potential of GSCs and increased their differentiation. miR-504 overexpression did not induce cell death in the GSCs as determined by trypan blue staining (data not shown).

In addition, overexpression of miR-504 also decreased GSC migration (Fig. 3E) and the expression of the mesenchymal markers CTGF, fibronectin 1 (FN), and YKL-40 (Fig. 3F). These results demonstrate that miR-504 acts as a negative regulator of the stemness, mesenchymal phenotypes, and migration of GSCs.

We further examined the effects of miR-504 overexpression on the tumorigenic capacity of GSCs in vivo. For these experiments we employed GSC-1 transduced with lentivirus vectors expressing pre-miR-504. Intracranial implantation of these cells into immunocompromised mice resulted in significantly smaller xenografts $(4.35 \pm$ $\left.1.33 \mathrm{~mm}^{2} ; n=5\right)$ compared with those expressing a control pre-miR $\left(11.96 \pm 4.20 \mathrm{~mm}^{2} ; \quad N=5, \quad P<0.005\right)$ (Fig. 3G). In addition, overexpression of miR-504 increased the mean survival of tumor-bearing mice compared with the control miR group (66 days vs. 42 days, $P<0.0001, N=11 /$ group) (Fig. $3 \mathrm{H}$ ). These data demonstrate that overexpression of miR-504 in GSCs decreased their tumorigenicity which is in line with the favorable prognosis of patients with tumors that express high levels of this miR.

\section{Grb10 is a target of miR-504 and mediates the inhibitory effects of this miR on GSCs}

Bioinformatics analysis identified Grb10 as a potential target of miR-504 which was also recently reported as a validated one $^{39}$. Using the Grb10 $3^{\prime}$-UTR- tagged to luciferase, we demonstrated a direct targeting of Grb10 by miR-504 (Fig. 4A). We then analyzed the expression of Grb10 in hNSCs and GSCs $(N=10)$ and found that this gene was highly expressed in GSCs compared to hNSCs (Fig. 4B). Using TCGA we analyzed the expression of Grb10 in various subtypes of glioma and found that the expression of Grb10 was significantly higher in GBM compared with low-grade tumors as determined by histology criteria (Fig. 4C) and the WHO grade (Fig. 4D). In addition, analysis of methylation glioma subtypes demonstrated that Grb10 was highly expressed in the 


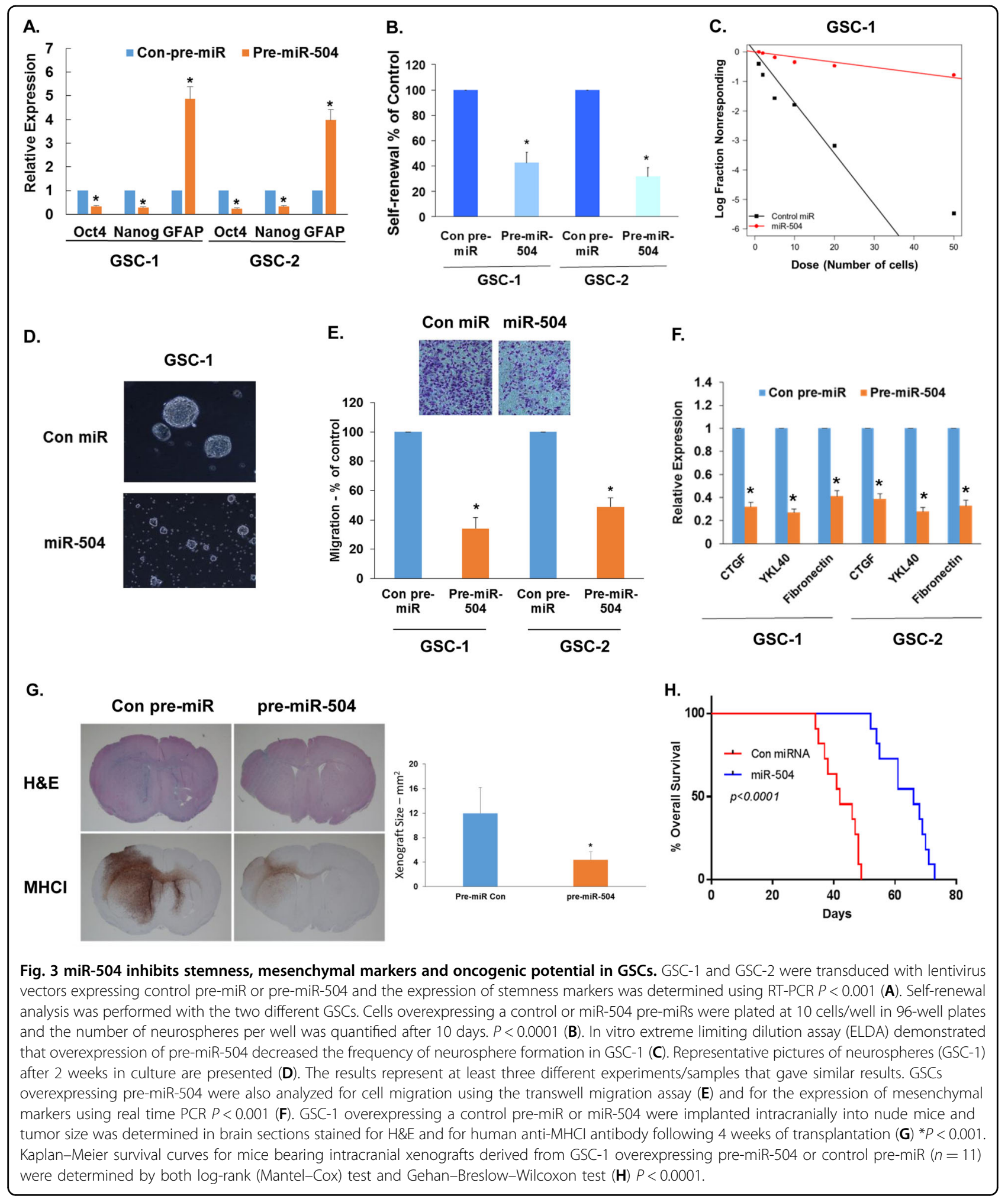

mesenchymal subtype and exhibited lowest expression in the G-CIMP high tumors (Fig. 4E). Kaplan-Meier analysis (Fig. 4F) shows that the quarter of patients with highest expression of Grb10 have the worst survival outcomes (log-rank $P=6.39 \mathrm{e}-12$ overall; log-rank $P=5.13 \mathrm{e}-11$, $6.15 \mathrm{e}-07,2 \mathrm{e}-05, \mathrm{Q} 4$ vs. Q1-3, respectively).

Dichotomizing miR-504 at the 25th and Grb10 at the 75th percentiles, demonstrates that the low/high pattern 


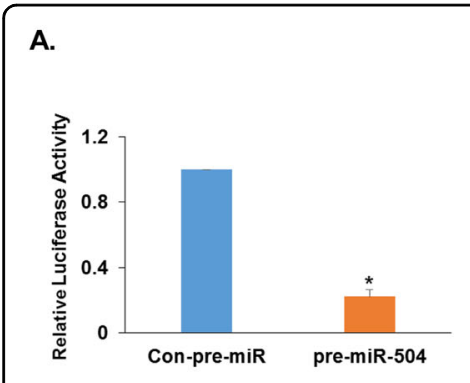

E.
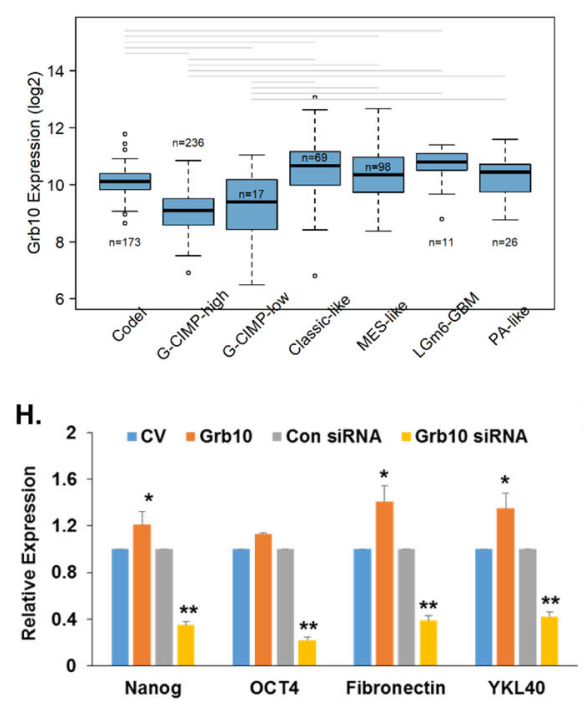
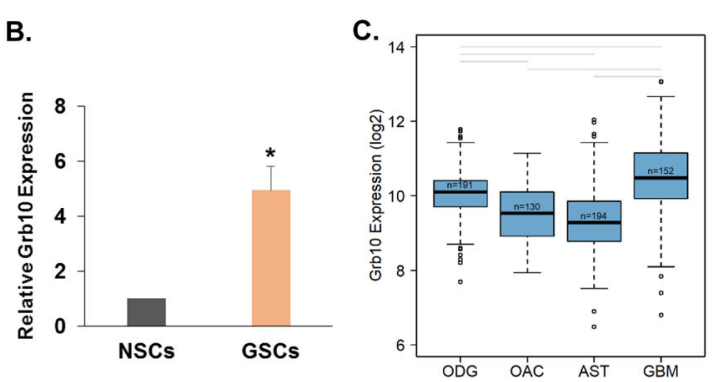

D.

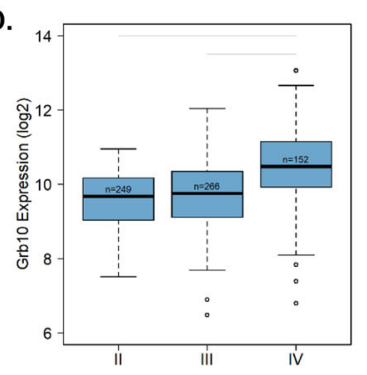

F.

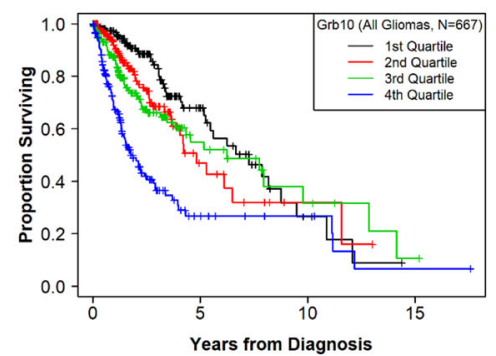

I.

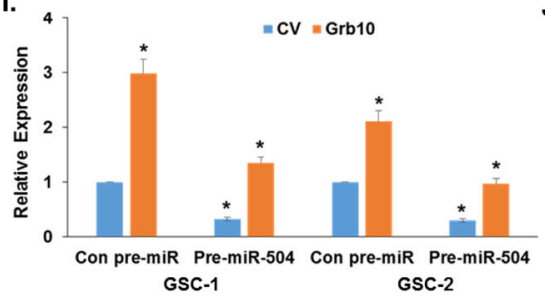

G.

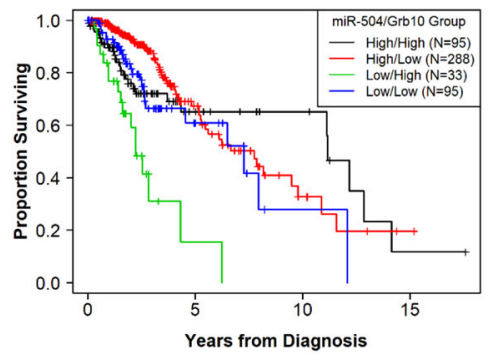

J.

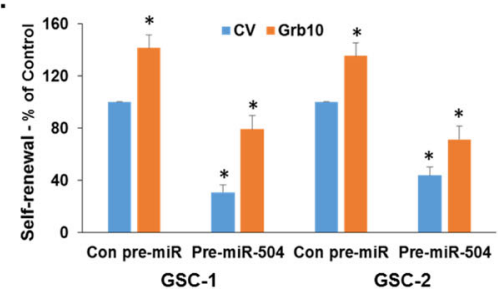

Fig. 4 Targeting of Grb10 mediates the anti-tumor effects of miR-504. The direct targeting of Grb10 by miR-504 was determined using luciferase assay of the Grb10 3'-UTR luciferase plasmid $P<0.001$ (A). The expression of Grb10 was determined in hNSCs $(N=4)$ and GSCs $(N=11)$ by RT-PCR $P$ $<0.01$ (B). The expression of Grb10 in the different subtypes of glioma was determined using data from TCGA. Boxplots of Grb10 expression are shown on a log-2 scale by histology (WHO2007 criteria); (ODG oligodendroglioma, OAC oligoastrocytoma, AST astrocytoma, GBM glioblastoma) (C), by WHO Grade (D), and by methylation subclass (E). Gray lines indicate a significant difference between groups (post hoc $t$ test, $P<0.05$ ). A Kaplan-Meier plot $(\mathbf{F})$ shows that the quarter of patients with highest expression of Grb10 have the worst survival outcomes (log-rank $P=6.39 \mathrm{e}-12$ overall; log-rank $P=5.13 \mathrm{e}-11,6.15 \mathrm{e}-07,2 \mathrm{e}-05$, Q4 vs. Q1-3). Overall survival plotted according to high/low expression of miR-504 and Grb10 dichotomized at 25th percentile for miR-504 and 75th percentile for Grb10 to provide a better separation. log-rank $P=1 \mathrm{e}-04$ (G). Median survival is indicated on the graphs. GSC-1 cells were transduced with lentivirus vectors expressing a control vector, Grb10, a control shRNA or Grb10 shRNA, and the expression of mesenchymal and stemness markers was analyzed using RT-PCR $(\mathbf{H}){ }^{*} P<0.005$; ${ }^{* *} P<0.01$. The role of Grb10 in miR-504 effects on Nanog expression (I) and self-renewal (J) was analyzed in GSCs transduced with lentivirus expressing miR-504 with and without Grb10 lacking the 3'UTR. ${ }^{*} P<0.001$. The results represent at least three different experiments that gave similar results.

of miR-504 and Grb10, respectively, more clearly identifies a subset of patients with poor overall survival compared to each expression alone (Fig. 4G, log-rank $P=2.67 \mathrm{e}-8$ overall; log-rank $P=0.0012,2.89 \mathrm{e}-12$, 0.0003 , low/high vs. high/high, high/low and low/low, respectively).

We then examined the role of Grb10 in GSC functions and demonstrated that its overexpression in GSCs increased (Fig. 4H), while its silencing decreased (Fig. 4H) the stemness and mesenchymal phenotypes of GSCs, similar to the effects of miR-504. Overexpression of a Grb10 plasmid lacking 3'-UTR induced a modest upregulation of self-renewal of the GSCs and partially abrogated the inhibitory effect of miR-504 on the mesenchymal phenotype (Fig. 4I) and the self-renewal (Fig. 4J) of the treated GSCs. These results demonstrate that targeting Grb10 by miR-504 mediates at least in part the inhibitory effects of miR-504 on the stemness and mesenchymal phenotypes of GSCs.

\section{Overexpression of miR-504 in GSCs promotes M1 (pro- inflammatory) phenotypes of cocultured microglial cells}

Glioma cells and GSCs have been demonstrated to induce polarization of microglia toward the M2 phenotype (anti-inflammatory/pro-tumorigenic) by the secretion of cytokines and EV-derived miRNAs ${ }^{23,40,41}$. 


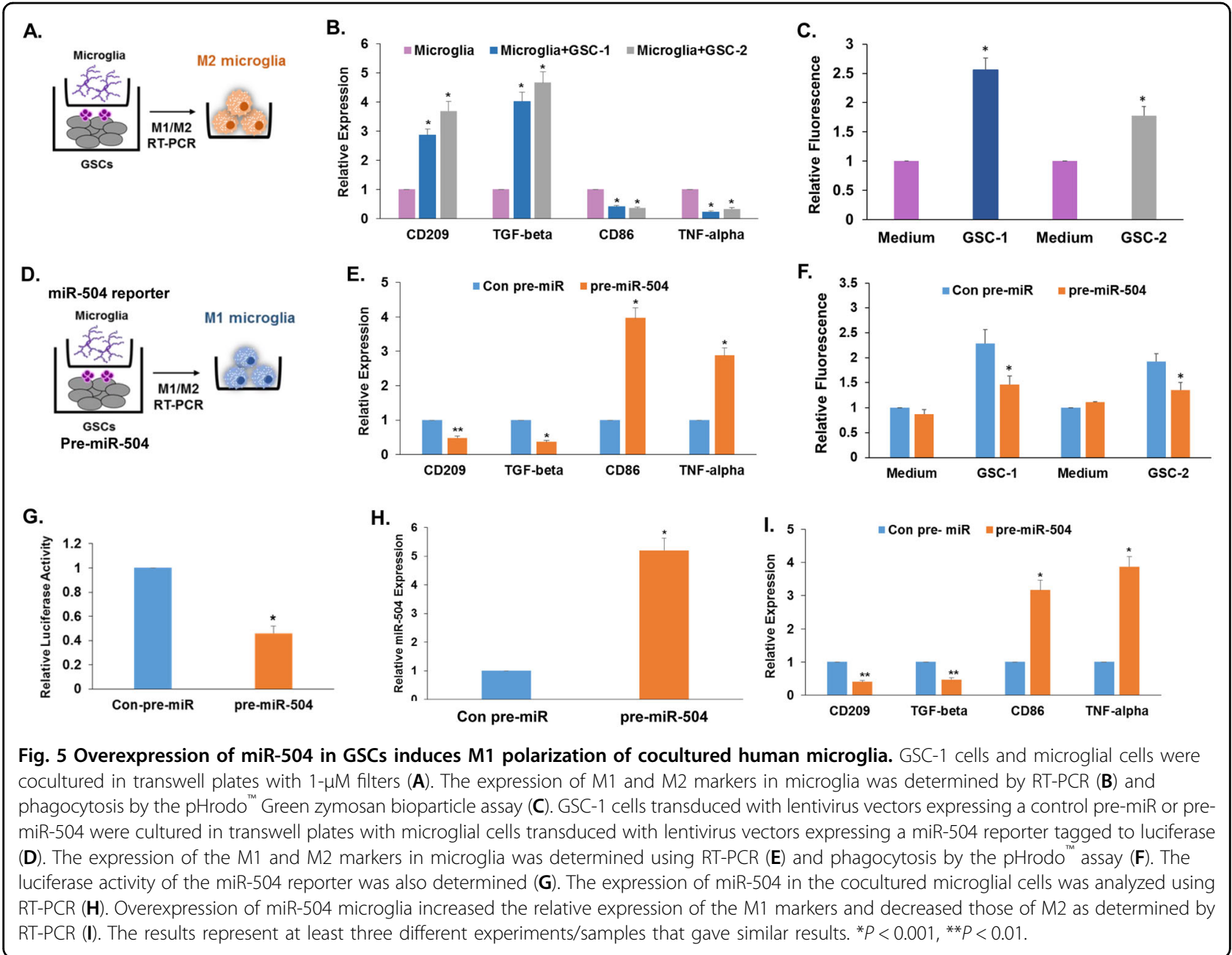

In addition, we recently reported that EVs can deliver exogenous miRNAs both in vitro and in vivo ${ }^{31,36}$. We therefore hypothesized that the overexpressed miR-504 in GSCs can be transferred to neighboring cells and therefore affects not only the oncogenic functions of the GSCs but also their interactions with neighboring cells such as microglia.

For these studies we employed co-cultures of microglial cells and GSCs overexpressing pre-miR-504 or a control pre-miR in transwell plates with $1-\mu \mathrm{m}$ filters that allow only the transfer of soluble factors and EVs as described in Fig. 5A. Coculturing of microglia with control GSCs or cells transduced with lentivirus expressing a control pre$\mathrm{miR}$ (Fig. 5A, D) resulted in a relative increased expression of the M2-associated markers, CD209 and TGF- $\beta$ and in the decreased expression of CD86 and TNF- $\alpha$ (Fig. $5 \mathrm{~B}, \mathrm{E})$. GSC coculturing also increased the phagocytosis of microglia cells (Fig. 5C). In contrast, transduction of the GSCs with pre-miR-504 (Fig. 5D) decreased the expression of the M2-associated markers, increased the expression of CD86 and TNF- $\alpha$ (Fig. 5E) and decreased phagocytosis (Fig. 5F). To verify that miR-504 was delivered by the GSCs to the cocultured microglial cells, we employed a miR-504 reporter tagged to luciferase that allows the quantification of the transferred miRNA as was previously reported ${ }^{33}$. Microglial cells were transduced with a lentivirus vector expressing the miR-504-luciferase reporter and the GSCs were transduced with lentivirus vector expressing either control pre-miR or pre-miR-504 (Fig. 5D). As presented in Fig. 5G, microglia that were cocultured with GSCs overexpressing pre-miR-504 exhibited decreased luciferase activity indicating that miR-504 was transferred by the cocultured GSCs. These results indicate that miR-504 was transferred by GSCs to cocultured microglial cells. We further analyzed the expression of miR-504 in the cocultured microglial cells and found that they expressed high levels of this miR compared with microglia cocultured with GSCs expressing a control miR (Fig. 5H). Finally, we demonstrated that overexpression of miR-504 in microglial cells upregulated the relative expression of the M1 markers CD86 and TNF- $\alpha$ (Fig. 5I). 


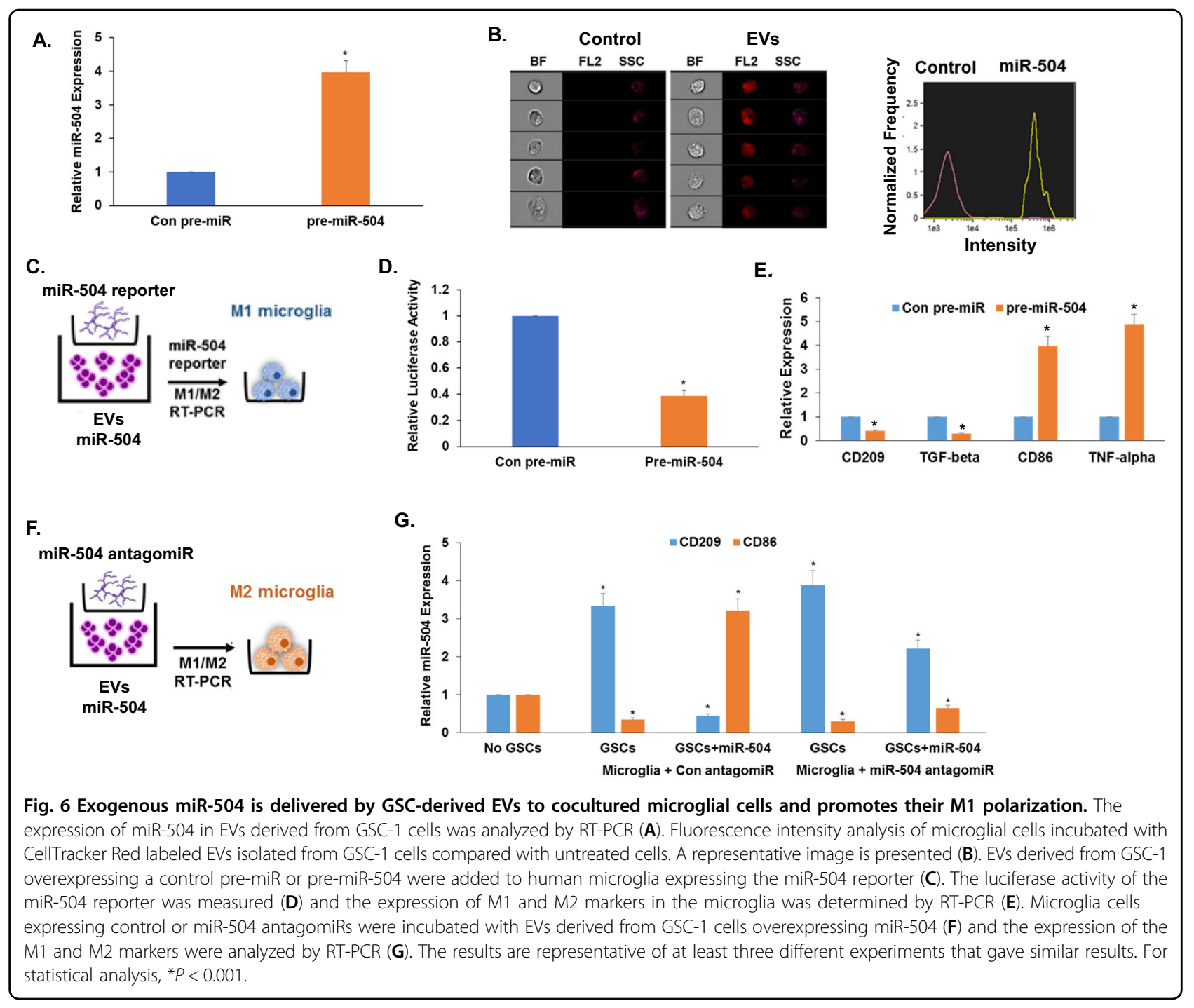

\section{EV-associated miR-504 derived from GSCs induces microglia M1 phenotypes}

EV-associated miRNAs are implicated in the cross-talk of GSCs and microglia ${ }^{33,35}$. To determine the role of EVs in the delivery of miR-504 to microglial cells, we first analyzed the expression of miR-504 in EVs derived from GSC-1 that were transduced with lentivirus vectors expressing control pre-miR or pre-miR-504. EVs were isolated from GSC-1 cultures using differential ultracentrifugation as previously described ${ }^{35}$ and were analyzed for the expression of CD63, CD81, and CD9 (Fig. S3B). The amount of the secreted EVs was determined using ELISA of CD63 antibodies and was found to be comparable in GSC-1 overexpressing con-miR or miR504 (data not shown). We found that EVs isolated from GSC-1 overexpressing miR-504 expressed significantly higher levels of miR-504 compared with EVs isolated from GSC-1 expressing a control pre-miR (Figs. 6A, S3C).
We next demonstrated the transfer of EVs from GSC-1 to microglial cells using ImageStreamX analysis. In these studies, GSC-1 derived EVs labeled with CellTracker Red were added to microglial cells and their fluorescence was determined $12 \mathrm{~h}$ later. The EVs were efficiently internalized and accumulated in the microglial cells (Fig. 6B). Incubation of microglial cells that express the miR-504 reporter with EVs that were isolated from GSC-1 expressing either the con-miR or miR-504 (Fig. 6C) demonstrated the functional delivery of the miR-504 to the microglial cells (Fig. 6D), which resulted in decreased expression of CD209 and TGF- $\beta$, and increased expression of CD86 and TNF- $\alpha$ (Fig. 6E), similar to our observations with cocultured GSCs (Fig. 5D). To demonstrate that the delivered miR-504 mediated the effects of the GSC-1 derived EVs, we examined the effects of EVs isolated from GSC-1 overexpressing miR-504 on the differentiation of microglial cells transfected with a miR-504 
antagomir (Fig. 6F) and found that these effects were markedly abrogated, whereas, no significant inhibition was observed in microglia transfected with a control antagomir (Fig. 6G). These results indicate that the transfer of miR-504 by GSC-derived EVs mediated, at least partly, the increased M1 phenotypes of the microglial cells induced by the cocultured GSCs.

\section{Discussion}

GBM is one of the most aggressive, infiltrative and incurable tumors with an average patient survival of around 14-16 months ${ }^{1,2}$. GBM therapy resistance and recurrence are primarily attributed to the existence of $\mathrm{GSCs}^{8-10}$. Therefore, targeting these cells is an essential component of any successful therapeutic approach. Recent studies demonstrated that the mesenchymal differentiation of GBM is associated with acquisition of stemness characteristics, tumor aggressiveness, therapy resistance, and poor clinical outcome ${ }^{16-18,42}$. Thus, deciphering the mechanisms underlying the mesenchymal differentiation of GSCs is essential for identifying novel therapeutic targets and improving patient survival.

miRNAs have emerged as attractive therapeutic targets due to their critical roles in the regulation of major cell processes such as cell proliferation, stemness, and apoptosis that are key components in cancer initiation and progression $^{29-31,43}$. In addition, specific miRNAs have been implicated in controlling the mesenchymal differentiation of tumor cells ${ }^{26,44,45}$.

Using miRNA microarray analysis of GSCs in comparison to hNSCs, we identified 85 miRNAs that were significantly altered in the GSCs compared with hNSCs. These miRNAs were found to be associated with wellknown tumorigenic pathways including cell cycle, cellular development, cellular growth and proliferation, cell-tocell signaling and interaction, and cell death and survival. These findings indicate that alterations in miRNA expression are associated with deregulation in pathways which contribute to the tumorigenic phenotypes of GSCs.

Additional differences in miRNA expression between GSCs and hNSCs were also associated with the EMT process and included pathways regulating $\operatorname{Smad} 2 / 3$, Smad6/7 TGF $\beta 1^{46}$, and Dicer ${ }^{47}$, suggesting that the expression of specific miRNAs in GSCs regulate their own expression in parallel with the tumorigenic characteristics of these cells. Finally, other IPA networks identified ZEB2 and RUNX1, two major regulators of the EMT pathway ${ }^{7}$, as mainly enriched in GSCs.

One of the most downregulated miRNAs in GSCs compared to hNSCs was miR-504. We further found that miR-504 expression was significantly lower in GBM as compared to normal brains and exhibited a gradedependent expression. In addition, using the TCGA portal, we found that miR-504 expression was significantly increased in the G-CIMP high glioma and more generally in IDH-mutant GBM, whereas, it was considerably lower in the IDH-wt glioma classes including the mesenchymallike subtypes. In agreement with the lower expression of miR-504 in more high grade tumors and in the mesenchymal subtype, we found that overexpression of miR-504 inhibited the self-renewal and mesenchymal phenotypes of GSCs, Collectively, the current results highlight miR-504 as a potential tumor suppressor miRNA and as a negative regulator of the tumorigenicity of GBM and GSCs.

Our results of a role of miR-504 as a tumor suppressor in glioma and as an inhibitor of mesenchymal transformation are in agreement with recent publications ${ }^{48-52}$. The current studies present new data regarding the expression of miR-504 in patient-derived GSCs compared with NSCs and in specific subtypes of gliomas including patient survival data. In addition, the current studies focus on the functions of miR-504 in GSCs including their tumorgenicity using intracranial xenografts.

The role of miR-504 in oncogenic processes appears to be tumor dependent. Thus, in gastric cancer miR-504 expression was decreased by the tumor suppressor gene Trefoil factor 1 (TFF1) that leads to the activation of $\mathrm{p} 53^{53}$, whereas miR-504 had a dual function in oral squamous cell carcinoma ${ }^{54}$. Moreover, serum expression of miR-504 were demonstrated to differentiate between primary and metastatic brain tumors ${ }^{55}$, suggesting a role of this miR as a diagnostic marker and a mediator of the interaction of glioma and non-CNS cells.

The inhibitory effects of miR-504 on GSCs were at least partly mediated by Grb10, which was recently reported as a validated miR-504 target in vascular endothelial cells ${ }^{39}$. Grb10 is an imprinted gene that is differentially expressed from two promoters and in the brain it is paternally expressed $^{56}$. The role of Grb10 in tumorigenesis is just beginning to be understood ${ }^{57,58}$. Using TCGA analysis, we demonstrated that Grb10 was highly expressed in more aggressive glioma tumors and its expression was directly correlated with worse prognosis. In addition, overexpression of Grb10 in GSCs promoted their aggressiveness, whereas silencing exerted an opposite effect and abrogated the inhibitory effect of miR-504. Thus, our studies demonstrated the miR-504/Grb10 pathway as an important regulator of the stemness-EMT process in GSCs.

Additional targets of miR-504 were reported in glioma and a recent study reported that miR-504 inhibited EMT by targeting the Frizzled-7-mediated the Wnt- $\beta$-catenin pathway $^{51}$.

GSCs have been reported to promote the differentiation of microglia toward the M2/anti-inflammatory phenotype, an effect that is more pronounced in mesenchymal $\mathrm{GSCs}^{23,40,41}$. The M2 microglia phenotype in turn acts to 


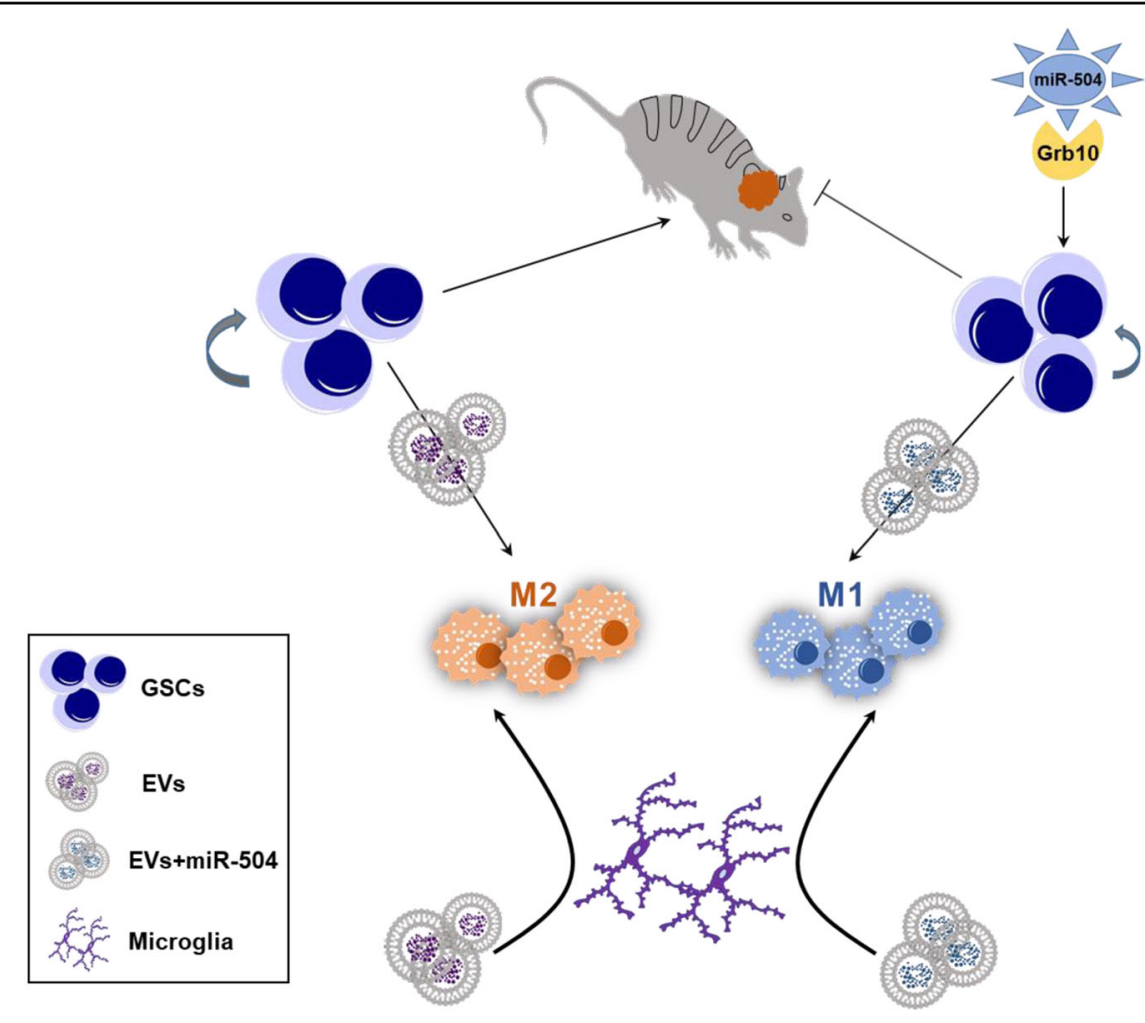

Fig. 7 A diagram summarizing the role miR-504 on GSCs and microglia polarization via delivery by EVs. The effects of miR-504 on GSCs and microglia polarization are depicted in this diagram.

support the migration and aggressiveness of the tumor cells and inhibition of anti-tumor immune response ${ }^{24,59,60}$. The cross talk between microglia and GSCs is mediated by secreted cytokines and EV-derived miRNAs ${ }^{23,40,41,61}$ In view of our recent reports that EVs can also deliver exogenous miRNA to neighboring cells $^{31,36}$, we hypothesized that overexpression of miR-504 in GSCs may be transferred to neighboring cells via EVs. Using a miR-504 reporter that can directly detect changes in miRNA levels, we found that GSCs overexpressing miR-504 delivered this miRNA to cocultured microglia via EVs. We also found that the increased relative expression of M2 markers induced by GSCs was abrogated in microglia co-cultured with GSCs overexpressing a miR-504 mimic.

The mechanisms by which the EV-derived miR-504 exerts its effects on the microglial cells are currently being studied. Since miR-504 decreased the stemness and mesenchymal differentiation of GSCs, it is possible that other factors or miRNAs that are secreted by the transduced GSCs can also contribute to the induction of this change in the microglial phenotype.

The mutual crosstalk between glioma cells and microglia highlights the importance of soluble factors as potential therapeutic targets. Indeed, EV-associated miRNAs have been reported to play an important role in intercellular interactions in both physiological and pathological conditions ${ }^{62,63}$. In addition, overexpressed miRNA mimics have been also reported to be delivered by EVs to neighboring cells ${ }^{36,39}$. Our data indicate that overexpressing miR-504 in GSCs affects not only the functions of the tumor cells but also the tumor-promoting activity of microglia and probably macrophages in the tumor microenvironment, thereby amplifying the therapeutic effect of miR-504 (Fig. 7).

\section{Conclusions}

The acquisition of mesenchymal phenotypes has been associated with increased stemness, infiltration, and aggressive phenotypes in GSCs ${ }^{64}$. Therefore, identifying therapeutic targets that can interfere with this process is of utmost importance. Performing comparative analyses of hNSCs and GSCs, we identified novel miRNAs and potential target networks that are associated with the stemness and mesenchymal transit of GSCs. miR-504 is downregulated in GSCs and exerts inhibitory effects on the functions of these cells via the targeting of Grb10 that acts as an oncogene in GBM and GSCs. Importantly, the 
overexpression of miR-504 in GSCs not only inhibits the tumorigenic potential of GSCs in vitro and in vivo but can be also transferred to microglial calls and promote their M1 polarization. Thus, the antitumor effects of RNA-based therapy in cancer cells can further exert a bystander effect on the tumor microenvironment via $\mathrm{EV}$ delivery.

\section{Acknowledgements}

We thank Dr. Hae Kyung Lee and Susan Finniss for their technical support and Susan McPhee for her diligent editing of the manuscript. This work was supported by the William and Karen Davidson Fund, Hermelin Brain Tumor Center and by ICRF (C.B.).

\section{Author details}

${ }^{1}$ The Mina and Everard Goodman Faculty of Life Sciences, Bar-llan University, Ramat-Gan, Israel. '2Davidson Laboratory of Cell Signaling and Tumorigenesis, Hermelin Brain Tumor Center, Department of Neurosurgery, Henry Ford Hospital, Detroit,

MI, USA. ${ }^{3}$ Department of Neurosurgery, Sheba Medical Center, Henry Ford Hospital, Detroit, MI, USA. ${ }^{4}$ Department of Public Health Sciences, Henry Ford Hospital, Detroit, MI, USA

\section{Data availability}

Data are available in the Supplementary files.

\section{Ethics approval and consent to participate}

All human materials were used in accordance with the policies of the institutional review board at Henry Ford Hospital, Detroit, MI.

\section{Conflict of interest}

The authors declare that they have no conflict of interest.

\section{Publisher's note}

Springer Nature remains neutral with regard to jurisdictional claims in published maps and institutional affiliations.

Supplementary Information accompanies this paper at (https://doi.org/ 10.1038/s41419-020-03088-3).

Received: 5 December 2019 Revised: 13 August 2020 Accepted: 24 August 2020

Published online: 22 October 2020

\section{References}

1. Furnari, F. B. et al. Malignant astrocytic glioma: genetics, biology, and paths to treatment. Genes Dev. 21, 2683-2710 (2007).

2. Desjardins, A. et al. Chemotherapy and novel therapeutic approaches in malignant gliomas. Front. Biosci. 10, 2645-2668 (2005).

3. Phillips, H. S. et al. Molecular subclasses of high-grade glioma predict prognosis, delineate a pattern of disease progression, and resemble stages in neurogenesis. Cancer Cell 9, 157-173 (2006).

4. Verhaak, R. G. W. et al. Integrated genomic analysis identifies clinically relevant subtypes of glioblastoma characterized by abnormalities in PDGFRA, IDH1, EGFR, and NF1. Cancer Cell 17, 98-110 (2010).

5. Ceccarelli, M. et al. Molecular profiling reveals biologically discrete subsets and pathways of progression in diffuse glioma. Cell 164, 550-563 (2016).

6. Brennan, C. W. et al. The somatic genomic landscape of glioblastoma. Cell 155, 462-477 (2013).

7. Carro, M. S. et al. The transcriptional network for mesenchymal transformation of brain tumours. Nature 463, 318-325 (2010).

8. Singh, S. K. et al. Identification of a cancer stem cell in human brain tumors. Cancer Res. 63, 5821-5828 (2003).

9. Vescovi, A. L., Galli, R. \& Reynolds, B. A. Brain tumour stem cells. Nat. Rev. Cancer 6, 425-436 (2006).
10. Lathia, J. D., Mack, S. C., Mulkearns-Hubert, E. E., Valentim, C. L. L. \& Rich, J. N. Cancer stem cells in glioblastoma. Genes Dev. 29, 1203-1217 (2015).

11. Lim, D. A. et al. Relationship of glioblastoma multiforme to neural stem cell regions predicts invasive and multifocal tumor phenotype. Neuro. Oncol. $\mathbf{9}$, 424-429 (2007).

12. Matarredona, E. R. \& Pastor, A. M. Neural stem cells of the subventricular zone as the origin of human glioblastoma stem cells. Therapeutic implications. Front. Oncol. 9, 779-824 (2019).

13. Nieto, M. A., Huang, R. Y. Y. J., Jackson, R. A. A. \& Thiery, J. P. P. EMT: 2016. Cell 166, 21-45 (2016).

14. Tso, C. L. et al. Primary glioblastomas express mesenchymal stem-like properties. Mol. Cancer Res. 4, 607-619 (2006).

15. Behnan, J., Finocchiaro, G. \& Hanna, G. The landscape of the mesenchymal signature in brain tumours. Brain 142, 847-866 (2019).

16. Fedele, M., Cerchia, L., Pegoraro, S., Sgarra, R. \& Manfioletti, G. Proneuralmesenchymal transition: Phenotypic plasticity to acquire multitherapy resistance in glioblastoma. Int. J. Mol. Sci. 20, 2746-2760 (2019).

17. Halliday, J. et al. In vivo radiation response of proneural glioma characterized by protective p53 transcriptional program and proneural-mesenchymal shift. Proc. Natl Acad. Sci. USA 111, 5248-5253 (2014).

18. Bhat, K. P. L. et al. Mesenchymal differentiation mediated by NF-KB promotes radiation resistance in glioblastoma. Cancer Cell 24, 331-346 (2013).

19. Quail, D. F. \& Joyce, J. A. The microenvironmental landscape of brain tumors Cancer Cell 31, 326-341 (2017).

20. Zanotto-Filho, A. et al. Inflammatory landscape of human brain tumors reveals an NFkB dependent cytokine pathway associated with mesenchymal glioblastoma. Cancer Lett. 390, 176-187 (2017).

21. Wang, Q. et al. Tumor evolution of glioma intrinsic gene expression subtype associates with immunological changes in the microenvironment. Cancer Cell 32, 42-56 (2017).

22. Chen, Z. \& Hambardzumyan, D. Immune microenvironment in glioblastoma subtypes. Front. Immunol. 9, 1004-1012 (2018).

23. Abels, E. R. et al. Glioblastoma-associated microglia reprogramming is mediated by functional transfer of extracellular miR-21. Cell Rep. 28, 3105-3119.e7 (2019).

24. Hambardzumyan, D., Gutmann, D. H. \& Kettenmann, H. The role of microglia and macrophages in glioma maintenance and progression. Nat. Neurosci. 19. 20-27 (2015).

25. Godlewski, J., Krichevsky, A. M., Johnson, M. D., Chiocca, E. A. \& Bronisz, A Belonging to a network-microRNAs, extracellular vesicles, and the glioblastoma microenvironment. Neuro Oncol. 17, 652-662 (2015).

26. Møller, H. G. et al. A systematic review of MicroRNA in glioblastoma multiforme: Micro-modulators in the mesenchymal mode of migration and invasion. Mol. Neurobiol. 47, 131-144 (2013).

27. Liu, C. \& Tang, D. G. MicroRNA regulation of cancer stem cells. Cancer Res. $\mathbf{7 1}$ 5950-5954 (2011).

28. Bier, A. et al. MicroRNA-137 is downregulated in glioblastoma and inhibits the stemness of glioma stem cells by targeting RTVP-1. Oncotarget 4, 665-676 (2013).

29. Lee, H. K et al. MicroRNA-145 is downregulated in glial tumors and regulates glioma cell migration by targeting connective tissue growth factor. PLOS ONE 8, e54652 (2013).

30. Giladi, N. D. et al. RTVP-1 promotes mesenchymal transformation of glioma via a STAT-3/IL-6-dependent positive feedback loop. Oncotarget 6, 22680-22697 (2015).

31. Lee, H. K. et al. Mesenchymal stem cells deliver synthetic microRNA mimics to glioma cells and glioma stem cells and inhibit their cell migration and selfrenewal. Oncotarget 4, 346-361 (2013).

32. Jiang, W. et al. Repurposing phenformin for the targeting of glioma stem cells and the treatment of glioblastoma. Oncotarget 35, 56456-56470 (2016).

33. Quartararo, C. E., Reznik, E., deCarvalho, A. C., Mikkelsen, T. \& Stockwell, B. R. High-throughput screening of patient-derived cultures reveals potential for precision medicine in glioblastoma. ACS Med. Chem. Lett. 6, 948-952 (2015).

34. Ziv-Av, A et al. RTVP-1 regulates glioma cell migration and invasion via interaction with N-WASP and hnRNPK. Oncotarget 6, 19826-19840 (2015).

35. Bier, A et al. Placenta-derived mesenchymal stromal cells and their exosomes exert therapeutic effects in Duchenne muscular dystrophy. Biomaterials 174, 67-78 (2018).

36. Lee, H. K., Finniss, S., Cazacu, S., Xiang, C. \& Brodie, C. Mesenchymal stem cells deliver exogenous miRNAs to neural cells and induce their differentiation and glutamate transporter expression. Stem Cells Dev. 23, 2851-2861 (2014). 
37. Brat, D. J. et al. Comprehensive, integrative genomic analysis of diffuse lowergrade gliomas. N. Engl. J. Med. 372, 2481-2498 (2015).

38. Li, B. \& Dewey, C. N. RSEM: accurate transcript quantification from RNA-Seq data with or without a reference genome. BMC Bioinform. 12, 323-339 (2011).

39. Reddy, M. A. et al. Regulation of vascular smooth muscle cell dysfunction under diabetic conditions by MIR-504. Arterioscler. Thromb. Vasc. Biol. 36 864-873 (2016).

40. De Vrij, J. et al. Glioblastoma-derived extracellular vesicles modify the phenotype of monocytic cells. Int. J. Cancer 137, 1630-1642 (2015).

41. Van Der Vos, K. E. et al. Directly visualized glioblastoma-derived extracellular vesicles transfer RNA to microglia/macrophages in the brain. Neuro Oncol. 18 58-69 (2016).

42. Li, G. et al. ALDH1A3 induces mesenchymal differentiation and serves as a predictor for survival in glioblastoma. Cell Death Dis. 9, 1190-1201 (2018).

43. Rynkeviciene, R. et al. Non-coding RNAs in glioma. Cancers 22, 11-46 (2018).

44. Tang, J. et al. Molecular mechanisms of microRNAs in regulating epithelialmesenchymal transitions in human cancers. Cancer Lett. 371, 301-313 (2016).

45. Hao, J. et al. MicroRNA control of epithelial-mesenchymal transition in cancer stem cells. Int. J. Cancer 135, 1019-1027 (2014).

46. Han, J., Alvarez-Breckenridge, C. A., Wang, Q. E. \& Yu, J. TGF- $\beta$ signaling and its targeting for glioma treatment. Am. J. Cancer Res. 5, 945-955 (2015).

47. Martello, G. et al. A microRNA targeting dicer for metastasis control. Cell 141, 1195-1207 (2010).

48. Guan, Y. et al. Downregulation of microRNA-504 is associated with poor prognosis in high-grade glioma. Int. J. Clin. Exp. Pathol. 8, 727-734 (2015).

49. Cui, R., Guan, Y., Sun, C., Chen, L., Bao, Y., Li, G., Qiu, B., Meng, X., Pang, C. \& Wang, Y. A tumor-suppressive microRNA, miR-504, inhibits cell proliferation and promotes apoptosis by targeting FOXP1 in human glioma. Cancer Lett. 28, 1-11 (2016)

50. Seo, J., Jin, D., Choi, C. H. \& Lee, H. Integration of microRNA, mRNA, and protein expression data for the identification of cancer-related microRNAs. Plos ONE $\mathbf{5}$ e0168412 (2017).

51. Liu, Q., Guan, Y., Li, Z., Wang, Y., Liu, Y., Cui, R. \& Wang, Y. miR-504 suppresses mesenchymal phenotype of glioblastoma by directly targeting the FZD7mediated Wnt- $\beta$-catenin pathway. Exp. Clin. Cancer Res. 16, 358-376 (2019).
52. Ma, X., Yoshimoto, K., Guan, Y., Hata, N., Mizoguchi, M., Sagata, N., Murata, H. Kuga, D., Amano, T., Nakamizo, A. \& Sasaki, T. Associations between microRNA expression and mesenchymal marker gene expression in glioblastoma. Neuro Oncol. 14, 1153-1162 (2012).

53. Soutto, M. et al. TFF1 activates p53 through down-regulation of miR-504 in gastric cancer. Oncotarget 5, 5663-5673 (2014).

54. Yang, M. H. et al. Connective tissue growth factor modulates oral squamous cell carcinoma invasion by activating a miR-504/FOXP1 signalling. Oncogene 31, 2401-2411 (2012)

55. Jin, Z., Jin, R. H., Ma, C., Li, H. S. \& Xu, H. Y. Serum expression level of miR-504 can differentiate between glioblastoma multiforme and solitary brain metastasis of non-small cell lung carcinoma. J. BUON 22, 474-480 (2017).

56. Plasschaert, R. N. \& Bartolomei, M. S. Tissue-specific regulation and function of Grb10 during growth and neuronal commitment. Proc. Natl Acad. Sci. USA 112, 6841-6947 (2015).

57. Khan, M. I. et al. Proproliferative function of adaptor protein GRB10 in prostate carcinoma. FASEB J. 33, 3198-3211 (2019).

58. Mroue, R., Huang, B., Braunstein, S., Firestone, A. J. \& Nakamura, J. L. Monoallelic loss of the imprinted gene Grb10 promotes tumor formation in irradiated Nf1 +/- mice. PLoS Genet. 11, 1-25 (2015).

59. Wu, A. et al. Glioma cancer stem cells induce immunosuppressive macrophages/microglia. Neuro Oncol. 12, 1113-1125 (2010).

60. Li, W. \& Graeber, M. B. The molecular profile of microglia under the influence of glioma. Neuro Oncol. 14, 958-978 (2012).

61. Bronisz, A., Godlewski, J. \& Chiocca, E. A. Extracellular vesicles and microRNAs: their role in tumorigenicity and therapy for brain tumors. Cell. Mol. Neurobiol. 36, 361-376 (2016)

62. Fan, Q. et al. The emerging role of exosome-derived non-coding RNAs in cancer biology. Cancer Lett. 414, 107-115 (2018).

63. Nakano, I., Garnier, D., Minata, M. \& Rak, J. Extracellular vesicles in the biology of brain tumour stem cells - Implications for inter-cellular communication, therapy and biomarker development. Semin. Cell Dev. Biol. 40, 17-26 (2015).

64. Iser, I. C., Pereira, M. B., Lenz, G. \& Wink, M. R. The epithelial-to-mesenchymal transition like process in glioblastoma: an updated systematic review and in silico investigation. Med. Res. Rev. 37, 271-313 (2017). 